Camilla Köhnken

\title{
Beethoven-Auslegung zwischen Liszts »Deklamationsstil《 und Bülows »Vivisektionsversuchen«. Auf den Spuren Liszt'scher Interpretationsideale in Hans von Bülows instruktiver Edition der Klaviersonaten Beethovens
}

Zwei Sonaten spielten in Franz Liszts Kernrepertoire stets eine zentrale Rolle: die Klaviersonaten op. 27 Nr. 2 (»Mondschein«) und op. Io6 (»Hammerklavier«) von Ludwig van Beethoven. Während letztere von ihm überhaupt erstmals ins pianistische Repertoire eingeführt worden war, trug der erste Satz der Mondscheinsonate - das »Steckenpferd « seiner Virtuosenzeit - dazu bei, seinen Ruf als unvergleichlicher >Dramatiker auf der Bühne zu begründen.

Eine Episode aus seinen letzten Lebensjahren illustriert diese Qualität in seinem Spiel, die auf die Zuhörer einen tiefen Eindruck machte, der jedoch offensichtlich schwer in Worte zu fassen war: Alexander Siloti, ein russischer Liszt-Schüler in den Jahren I88I bis I886, hatte Anton Rubinstein - den vielleicht prominentesten Virtuosen und Beethoven-Spezialisten dieser Jahre - die Mondscheinsonate in einer Matinée im Leipziger Gewandhaus spielen hören und erzählte Liszt begeistert davon. Dessen Ehrgeiz wurde davon so angestachelt, dass er sich spontan der Unerfahrenheit einer Schülerin bediente, die das Stück trotz des >Verbots in den Unterricht gebracht hatte, um eine der letzten Demonstrationen seiner herausragenden Interpretation zu geben:

»Liebes Kind, man darf mir dieses Stück nicht bringen; ich erlaube nicht, daß man es spielt, weil es in der Jugend mein >Steckenpferd « gewesen ist, aber, da `wir $`$ heute in guter Stimmung sind, so werde ich Ihnen die Sonate vorspielen.«

Siloti beschreibt den Eindruck folgendermaßen:

"Als er kaum die Einleitungstriolen gespielt hatte, fühlte ich schon, daß ich in diesem Zimmer fast nicht mehr existierte; aber als nach 4 Takten das gis in der rechten Hand anfing, verstand ich nichts mehr. Dieses gis hob er nicht hervor, aber es war ein mir unbekannter Ton, welchen ich jetzt, nach 27 Jahren, noch deutlich höre. [...] Nach dieser Aufführung vergaß ich, daß ich vor zwei Stunden A. Rubinstein gehört hatte! Rubinstein, als Pianist, existierte nach Liszt's Spiel nicht mehr!«

Den eigentlichen Liszt-Klang beschreibt er dabei wie folgt: „Er hatte keinen sehr großen Ton, aber wenn er spielte, dann spielte der Klavierton gar keine Rolle mehr«.

Liszt reagierte auf den von ihm verursachten Aufruhr in einer typischen Mischung aus Eitelkeit und Abgeklärtheit: »Ja, wir verstehen es noch zu spielen. ${ }^{\mathrm{I}}$

1 Alexander Siloti: Meine Erinnerungen an Franz Liszt. Aus dem Russischen übertragen von Sophie Korsunska, in: Zeitschrift der internationalen Musikgesellschaft I4 (I9I2/I3), S. 294-318, hier S.305f. sowie S. 304 . 
Dieser Bericht ist in einem Aspekt besonders exemplarisch für die Bemühungen der Interpretationsforschung, Liszts eigenes Spiel möglichst genau zu rekonstruieren: jenem der ultimativen Vagheit. Der Leser erfährt zwar, dass der Eindruck ein so ungeheurer war, dass Siloti das erste gis der rechten Hand noch 27 Jahre später hörte. Was daran konkret aber so unvergesslich war - also auf welche Weise Liszt das gis nun genau anschlug - bleibt unklar. Nur dass es kein lauter Ton war, ist aus der emotionsgeladenen Beschreibung noch herauszufiltern.

Seine Interpretationsgrundsätze gab Liszt an seinen Schüler Hans von Bülow (I830I894) weiter, der in den Jahren I85I-I853 bei ihm studierte. Von Bülow gehörte damit zur ersten Schülergeneration in Weimar, der auch der von Liszt ebenfalls zeitlebens hochgeschätzte Carl Tausig angehörte. Bülow genoss von Anfang an eine Sonderstellung bei Liszt, der ihn neben dem Klavierunterricht oft auch zu seinen Engagements als Dirigent mitnahm.

»Sein Plan ist der: ich soll in Weimar ein Jahr bleiben und mich da vorzüglich einpauken - die neuen Werke von ihm, die Beethoven'schen größeren Sonaten, das Beste von Chopin, Schumann, kurz, mir ein Repertoire zu eigen machen, das nicht jeder, oder kein Pianist aufzuweisen im Stande sei ${ }^{2}{ }^{2}$

Entsprechend der eindrucksvollen Begabung und Arbeitskraft Hans von Bülows war seine lange Karriere als Musiker sehr facettenreich: Liszt charakterisierte seine Tätigkeiten I884 als »Virtuos, Docent, Dirigent, Kommentator, Propagandist - ja selbst als manchmal humoristisch gutgelaunter Journalist «. 3 Und wie Liszt beschäftigte sich Bülow sein ganzes Leben hindurch mit Beethoven, den er die »Centralsonne der modernen Tonwelt« nannte. ${ }^{4}$ Anfänglich spielte er die zum Standardrepertoire gehörenden Sonaten Pathétique, Mondscheinsonate und Appassionata, dann auch die mittleren und frühen und schließlich besonders oft die späten Sonaten.

Erstmals in der Geschichte des Solorezitals widmete er I864 in Leipzig einen ganzen Abend einem einzigen Komponisten - Beethoven - mit den Variationen F-Dur op. 34 und den Sonaten op. 8гa (»Les Adieux«), Ior und ro6. Solche Programme wurden jedoch von vielen Zeitgenossen, darunter auch dem ihm sonst gewogenen Eduard Hanslick, mit Unbehagen aufgenommen: »Indem Bülow sein Programm chronologisch ordnet, be-

Brief an den Vater vom 6. Juni I85ז, zit. nach Armin Raab: »Zentralsonne der modernen Tonwelt«. Bülows Beethoven-Verständnis, in: Beiträge zum Bülow-Kolloquium. Hans von Bülow - Leben, Wirken und Vermächtnis, hg. von Herta Müller und Verona Gerasch, Schweinfurt 1994 (Südthüringer Forschungen, Bd. 28), S. I80-I9I, hier S. I82.

3 Aus der Widmung des Bülow-Marsches von Liszt an seinen ehemaligen Schüler, zit. nach Hans-Joachim Hinrichsen: Musikalische Interpretation. Hans von Bülow, Stuttgart 1999 (Beihefte zum Archiv für Musikwissenschaft, Bd. 46), S. 15 .

4 Zit. nach ebd., S. 93. 
tont er die lehrhafte Absicht des Unternehmens. Eine Art Collegium über die Entwicklung Beethoven's in lauter Musikbeispielen. $\$ 5$

Dieser Antrieb zur Bildung des Publikums war ein durchgängiges und zentrales Element in Bülows musikalischem Leben und stellte ein wichtiges Bindeglied zwischen seinen diversen Tätigkeiten dar. Er führte parallel zu dem im englischsprachigen Raum gebräuchlichen Begriff >Recital den Begriff »Klavier-Vortrag « für seine Solo-Konzerte ein, was diesen publikumsbildenden Aspekt unterstrich und auch so aufgefasst wurde: »Bülow will durch seine Klavier-Konzerte (nicht unabsichtlich nennt er sie Klavier-Vorträge) vor allen Dingen belehrend wirken «. ${ }^{6}$

Dieser didaktische Impetus handelte ihm einige Kritik ein, besonders aus dem Lager seines pianistisch-pädagogischen Gegenpols Clara Schumann. Bülow unterstellte ihr sarkastisch, dass sie seine Beethoven-Aufführungen als »Vivisektionsversuche» wahrnahm.7 Tatsächlich tritt diese Auffassung in einer ihrer Tagebuchnotizen zutage: Der Eintrag stammt aus dem Jahr I884 und betrifft seine Beethoven-Proben mit dem Meininger Hoforchester in Frankfurt: »Er studirt ein, wie er spielt, zerpflückt und zergliedert Alles - das Herz hat nichts dabei zu thun. Alles der Kopf, der berechnet. ${ }^{8}$

Zusätzlich spiegelt der neugeprägte Begriff»Klavier-Vortrag« aber auch Bülows Anspruch wider, eine pianistische Darbietung nicht nur als ein bloßes Spiel auf dem Klavier zu präsentieren, sondern als ein »rhetorisch richtiges Clavierspie[1]«, 9 wie er es während seiner musikalischen Ausbildung bei Franz Liszt kennengelernt hatte.

"Als Frucht seiner Lehre ${ }^{\mathrm{IO}}$ widmete Hans von Bülow denn auch seinem Lehrer Franz Liszt den großangelegten »Interpretationsversuch« der Klaviersonaten Beethovens - die instruktive Ausgabe, an der er von I868 an arbeitete und die I87ı beim Verlag Cotta erschien. In diesem Projekt konnte er die Ergebnisse seiner intensiven Beschäftigung mit Beethovens Musik mit dem für ihn so wichtigen Bildungsgedanken besonders

5 Zit. nach Raab: »Zentralsonne der modernen Tonwelt«, S. I82.

6 I880 im Fachblatt der Klavierpädagogen Der Klavierlehrer, zit. nach Hinrichsen: Musikalische Interpretation, S. 236

7 Brief vom i2. Januar 1884 an Johannes Brahms, in: Hans von Bülow: Die Briefe an Johannes Brahms, hg. von Hans-Joachim Hinrichsen, Tutzing I994, S. 48.

8 Berthold Litzmann: Clara Schumann. Ein Künstlerleben nach Tagebüchern und Briefen, Bd.3: Clara Schumann und ihre Freunde, I856-I896, Leipzig I908, S. 448.

9 Carl Fuchs: Die Zukunft des musikalischen Vortrages und sein Ursprung. Studien im Sinne der Riemannischen Reform und zur Aufklärung des Unterschiedes zwischen antiker und musikalischer Rhythmik, Danzig I884, S. 23; vgl. auch Hinrichsen: Musikalische Interpretation, S. 236.

10 Sonaten und andere Werke für das Pianoforte von L. v. Beethoven in kritischer und instructiver Ausgabe mit erl. Anmerkungen für Lehrende und Lernende von Hans von Bülow, Stuttgart I884 [in Folge zitiert als BülowAusgabe], Vorwort, unpag. 
wirkungsvoll vereinen. ${ }^{\text {II }}$ Liszts Urteil über die Ausgabe lautete, sie »wiege an Belehrung ein Dutzend Konservatorien auf «. ${ }^{12}$ Clara Schumann hingegen verbot es ihren Schülern ganz, diese Ausgabe zu benutzen.

Tatsächlich nehmen die ausführlichen Kommentare am Fußende jeder Seite oft mehr Platz ein als der Notentext darüber. Man kann diese Erklärungen und Instruktionen in drei Kategorien aufteilen: praktisch-pianistische Kommentare zu Fingersätzen, Ausführungen von Ornamenten und Ähnlichem, sodann musikalische Erläuterungen zu Tempoaspekten, strukturanalytischen Fragen und Instrumentierungsvorschlägen und schließlich ästhetische Kommentare mit vielen Querverweisen zu anderen Werken Beethovens sowie zu dessen Einfluss auf spätere Komponisten.

Mit seiner »Editionsästhetik«strebte Bülow eine Integrität der Werke im Sinne eines ganz anderen Begriffs von >Werktreue an, als er heute gemeinhin benutzt wird: Die Interpretation des Notentextes wurde von ihm als eigenständige Kunstleistung angesehen, deren Ideal also keinesfalls mit >Texttreue gleichzusetzen ist. ${ }^{\mathrm{I3}}$ Heinrich Schenker trat am Anfang des 20. Jahrhunderts als prominentester und schärfster Kritiker der instruktiven Ausgaben und besonders der Beethoven-Ausgabe Bülows hervor, die er als »Herausgeberfrevel « bezeichnete. $^{\mathrm{I}}{ }^{4}$

Hans von Bülow bezieht sich in seinen Instruktionen zu den Sonaten ab op. 53 in fünf Fällen direkt auf Franz Liszt und greift in zahlreichen weiteren Fällen erkennbar Prinzipien Liszt'scher Aufführungspraxis auf, wenn auch ohne direkte Referenz. Letztere betreffen vor allem den wirkungsvolleren Vortrag bestimmter Stellen, der sich in Oktavverdoppelungen oder Änderungen von einfachen Trillern zu breiteren Tremoli ausdrückt. ${ }^{\text {I5 }}$

Der Liszt-Schüler Arthur Friedheim, welcher sich ebenfalls als Herausgeber betätigte, äußert im Vorwort seiner instruktiven Ausgabe der Chopin-Etüden eine sehr allgemein gehaltene Kritik an Bülows Ausgabe, die jedoch spezifisch auf die Überlieferung von Franz Liszts Interpretationsidealen anspielt: »Bülow's celebrated Beethoven edition [betrays], despite its many good points, in the main [...] to the initiated how little he had

11 Bände Nr. 4 und 5 der Cotta-Ausgabe (op.53-III und Variationen inklusive Diabelli-Variationen, Bagatellen, Polonaise und Rondo op. I29), später gab er noch die op.13, 26, 27/2 und 31/3 bei der Universal Edition Wien heraus (wovon wiederum ein Nachdruck bei Schirmer erschien). Wiedergabe nach Otto Leßmanns Nachruf auf Bülow in der Allgemeinen Musik-Zeitung vom r6. Februar I894, zit. nach Raab: »Zentralsonne der modernen Tonwelt«, S. I87.

13 Vgl. das Kapitel »Die Edition als Bearbeitung und Interpretation: Musikalische Praxis als ästhetisches System«, in: Hinrichsen: Musikalische Interpretation, S. I2I-204.

14 Zit. nach ebd., S. 205.

15 Vgl. Axel Schröter: »Der Name Beethoven ist heilig in der Kunst«. Studien zu Liszts Beethoven-Rezeption, Bd. I und 2, Sinzig I999 (Musik und Musikanschauung im I9. Jahrhundert. Studien und Quellen, Bd. 6), S. 29I und 3I7. 
learned, at the time, from Liszt. ${ }{ }^{6}$ In dieses Urteil spielt allerdings neben dem Eigeninteresse Friedheims als Herausgeber auch der Anspruch, selbst ein wahrer Liszt-Schüler zu sein.

Exemplarische Stellen im Vergleich der Ausgaben Liszts und von Bülows Schon am Anfang des ersten Satzes der Mondscheinsonate zeigt sich sowohl im Detail wie im größeren Überblick, dass man tatsächlich von Bülows Ansichten nicht direkt auf Liszts Überzeugungen schließen kann, zumindest wenn man Liszts eigene Ausgabe von I857 konsultiert: Während Liszt Beethovens Bezeichnung senza sordino (ohne Dämpfung, also mit durchgängigem >rechtem` Pedal) unverändert stehen lässt und sich auch im Folgenden aller Zusätze enthält, schreibt Bülow genau das Gegenteil - con sordini (Abbildung I).

Man könnte zunächst denken, dass Bülow mit con sordini hier die tatsächliche Abdämpfung, also die vom >linken Pedal gesteuerte Verschiebung meint, aber deren Einsatz wird schon mit der Angabe una corda in der Mitte des Notensystems abgedeckt. Außerdem fügt er zahlreiche kleingliedrige Pedalbezeichnungen hinzu, die erst in der Mitte des dritten Taktes beginnen - die ersten Takte will Bülow also >trocken $<$ das heißt mit Dämpfung gespielt wissen. Auch eine Instruktion am Fuß der Seite wird diesem Thema gewidmet: Dort genehmigt er zwar einen häufigeren Einsatz des Pedals als den eingezeichneten, warnt aber zugleich davor und auch vor der »zu wörtlichen «Auffassung der Originalangabe, also dem Verzicht auf jeglichen Pedalwechsel. ${ }^{\text {I7 }}$

Franz Liszt schreibt in seinem I853 veröffentlichten Brief über das Dirigieren, darin besonders auf Beethovens Spätwerk bezogen, diese Musik erfordere

"[...] einen Fortschritt in der Betonung, in der Rhythmisirung, in der Art gewisse Stellen im Detail zu phrasiren und zu deklamiren und Schatten und Licht im Ganzen zu zu [sic] vertheilen - mit einem Wort: einen Fortschritt im Stil der Ausführung selbst. [...] an vielen Stellen arbeitet die grobe Aufrechterhaltung des Taktes und jedes einzelnen Takttheiles $\mid \mathrm{I}, 2$, 3, 4 4 I, 2, 3, $4 \mid$ einem sinn- und verständnisvollen Ausdruck geradezu entgegen. Hier wie allerwärts, tödtet der Buchstabe den Geist«..$^{18}$

Die Schaffung größerer Sinnzusammenhänge war für Liszt immer ein zentraler Punkt für eine sinnvolle Interpretation, und dieses Ideal wird auch in den Unterrichtsprotokollen regelmäßig thematisiert. Allein sein oft dokumentiertes Beharren auf einer ruhigen Sitzhaltung am Klavier zielt auf diese Wahrung des Überblicks im Gegensatz zu einer kleingliedrigen Gestaltung aneinandergereihter Details ab, welche sich meist in einer

Arthur Friedheim: Frédéric Chopin. Etudes for the piano, New York I9I6, S. I.

18 Franz Liszt: Ein Brief über das Dirigieren. Eine Abwehr (1853), in: ders.: Streifzüge. Kritische, polemische und zeithistorische Essaps, hg. von Lina Ramann, Leipzig I882 (Gesammelte Schriften, Bd. 5), S. 227-232, hier S. 23I. 


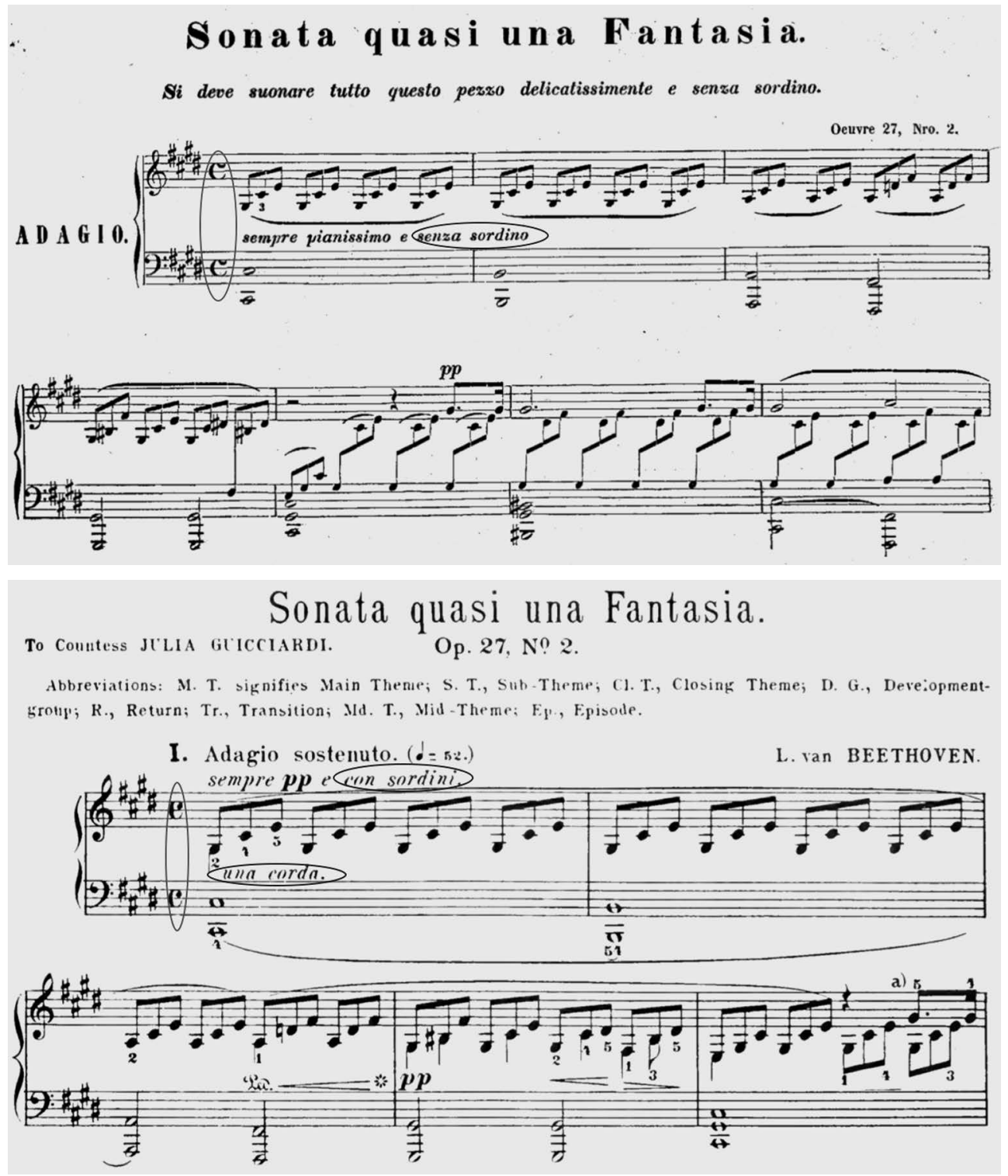

A в B IL D U N G 1 Liszt-Ausgabe, Ludwig Holle 1857 (oben) und Bülow-Ausgabe, Schirmer 1896 (unten)

übertrieben ausdrucksvollen und von unterstützenden Bewegungen begleiteten Körpersprache zeigt.

In Liszts Ausgabe sind, wie schon beschrieben, besonders die von ihm gesetzten Buchstaben zur Formgliederung interessant: Sie geben einen Anhaltspunkt für die Rekonstruktion seines Formverständnisses. Es lassen sich zwei verschiedene Arten der Einteilung voneinander unterscheiden: Die Markierung der kleineren periodischen Einheiten, entweder auf motivisch-thematischer oder auf harmonischer Ebene, und eine besonders interessante Gliederung in unterschiedlich lange Abschnitte, woraus sich 
Schlüsse über seine Gewichtung einzelner Passagen ziehen lassen. Dabei deutet die Schaffung kurzer Abschnitte auf eine von ihm postulierte besonders hohe Wichtigkeit dieser Stelle innerhalb der Struktur hin. Besonders lange Passagen, die er mit keinem Gliederungsbuchstaben unterbricht, unterstreichen hingegen sein Streben nach der Schaffung größerer Sinnzusammenhänge. ${ }^{\text {I9 }}$

Eine derartige Zusammenfassung einzelner Motive zu einer größeren Einheit oder sogar ganzer musikalischer Formteile zu einem noch längeren Entwicklungsbogen betrifft das Verhältnis einzelner Taktteile oder ganzer Takte zueinander. Dieses `Zusammenraffen ging in der praktischen Umsetzung meist mit einer Tempobeschleunigung einher, so wie umgekehrt bedeutsame Verbindungsstellen oder harmonisch wichtige Wendungen ein lokales Verlangsamen mit sich brachten. Die starke Verbindung zwischen Struktur und Tempo erklärt also die Vorwürfe der »Tempoverzerrung«, denen Interpretationen aus dem Liszt-Kreis oft ausgesetzt waren. ${ }^{20}$

Das Streben nach Verstärken und Präsentieren von Zusammenhängen konnte aber auch großformal den Übergang zwischen den Sätzen einer Sonate betreffen. Liszts als geschmack- und pietätlos empfundenes Aneinanderreihen von Sätzen aus verschiedenen Sonaten (wie in Hallés Bericht über die Kombination von Variationssatz der Sonate op. 26 und Finale der Mondscheinsonate ${ }^{2 \mathrm{I}}$ ) diente allerdings wohl eher dem Erreichen eines verblüffenden Effektes beim Publikum.

Aus Hans von Bülow Konzerten ist jedoch belegt, dass auch er bewusst und aus »wissenschaftlichen« Gründen Teile einer anderen Sonate derjenigen gegenüberstellte, die auf dem Programm stand - dies sollte dazu dienen, Verwandtschaften zwischen verschiedenen Stücken herauszustellen. ${ }^{22}$ Abgesehen von solchen spezifischen Vergleichszitaten schien Bülow auch sonst in seinen Beethoven-Darbietungen eine Praxis des Präludierens und Zwischenspielens zu pflegen: Er stellte Sonaten öfters kleine Improvisationen voran oder leitete »unmerklich aus einer Composition in die andere über «. ${ }^{23}$

19 Bezüglich »der Gruppierung mehrerer Einzelmotive zu einem thematischen Komplex«, siehe Schröter: »Der Name Beethoven ist heilig in der Kunst«, S. 309 .

20 Vgl. beispielsweise Ernst Rudorff: Aus den Tagen der Romantik. Bildnis einer deutschen Familie, Bd.3, hg. von Katja Schmidt-Wistoff, Frankfurt a. M. u. a. 2008 (Geschichte des Natur- und Umweltschutzes, Bd. 6), S. 86.

"I was very young and most impressionable, but still his tacking on the finale of the $\mathrm{C}$ sharp minor sonata (Beethoven's) to the variations of the one in A flat, Op.26, gave me a shock, in spite of the perfection with which both movements were played.« Nach Life and letters of Sir Charles Hallé, being an autobiography (I8I9-I860) with correspondence and diaries, hg. von C. E. Hallé und Marie Hallé, London I896, S.38.

22 Vgl. Raab: »Zentralsonne der modernen Tonwelt«, S. I84.

23 Nach einem Artikel aus der Berliner Tribüne von Ende Januar I879, zit. von Raab: »Zentralsonne der modernen Tonwelt«, S. I8 3 f. 
Eine weniger kontroverse Art der Herstellung von großformalen Zusammenhängen überliefert Amy Fay, eine amerikanische Schülerin Liszts, von einer seiner Aufführungen der Mondscheinsonate im Jahr I872. Dort beschränkte sich Bülow auf die zeitlichen Bezüge zwischen den Sätzen:

»Did I tell you how carried away with Bülow I was? He is magnificent, and just between Rubinstein and Tausig. [...] I wish you could have heard the Moonlight Sonata from him. One thing he does which is entirely peculiar to himself. He runs all the movements of a sonata together, instead of pausing between. It pleased me very much, as it gives a unity of effect, and seems to make each movement beget the succeeding one. $\ll^{24}$

Fays Bericht kann man in Bülows Ausgabe der späten Sonaten nachvollziehen: Er fordert in verschiedenen Instruktionen zu den Sonaten op. IOI, Iog und IIo genau dieses Prinzip des zyklischen Zusammenhalts der einzelnen Sätze untereinander. So begründet Bülow in der Sonate op. Ior einen Übergang zwischen den Sätzen ohne jegliche Pause mit dem psychologischen Effekt, der erst durch die einander unmittelbar gegenübergestellten Kontraste zur Geltung käme. ${ }^{25}$ Besonders bei op. Iro legt er Wert auf eine organische und fast unmerkliche Überleitung vom zweiten in den dritten (langsamen) Satz. Außerdem empfiehlt er die Herstellung einer zeitlichen Entsprechung zwischen der Sechzehntelbegleitung des Arioso und dem Achtelpuls der Fuge. ${ }^{26}$

Liszt scheint den ersten Satz der Mondscheinsonate ziemlich langsam gespielt zu haben. Dafür spricht einerseits seine Änderung des originalen Alla-Breve-Takt in einen 4/4-Takt, wie er dann auch von Bülow übernommen wurde. Darüber hinaus gibt es ein Zeugnis von August Stradal, einem weiteren Schüler und Sekretär der letzten Jahre, welcher Liszts Tempo mit der Angabe Larghetto und Viertel $=58$ genau definierte. Stradal führt weiter aus, dass Liszt die Triolen in der rechten Hand ganz gleichmäßig und tonlos, ohne Anschwellungen vorgetragen habe. Die Sechzehntel der Punktierung sei dabei immer etwas länger gehalten worden, sodass sie fast triolisch geklungen habe. Dadurch hätten die Noten etwas Schweres, nach pesante Klingendes bekommen. ${ }^{27}$

Es sind drei Aufnahmen von direkten Liszt-Schülern überliefert: von Arthur Friedheim (I9I2), Emil Sauer (I923) und Frederic Lamond (I926). ${ }^{28}$ Lamond spielt das zügigste Tempo, etwa Viertel $=74$, und auch Sauer bewegt sich mit ungefähr Viertel $=72 \mathrm{im}$ gleichen Tempobereich. Friedheim hingegen interpretiert den Satz deutlich langsamer:

24 Amy Fay: Music-Study in Germany. From the Home Correspondence of Amy Fay, Chicago 9ı886, S. I68.

25 Vgl. Bülow-Ausgabe, Bd. 5, S. 6.

26 Vgl. Bülow-Ausgabe, Bd.5, S. IIо.

27 Vgl. August Stradal: Erinnerungen an Franz Liszt, Bern I929, S. 87.

28 Emil Sauer: www.youtube.com/watch?v=DJfnBYooJuM; Frederic Lamond: www.youtube.com/watch? v=daQFLCSc9Ok; Arthur Friedheim: www.youtube.com/watch?v=-9 Diu ${ }_{9}$ nrVJc (letzter Zugriff jeweils 27. Juni 20I8). 
Er spielt tatsächlich (mit den üblichen Abweichungen) das von Stradal angegebene Liszt'sche Tempo von Viertel $=58$.

Lamond führt dabei die Sechzehntel der Oberstimme ziemlich genau im Tempo aus, während die triolische Gestaltung bei Friedheim und besonders bei Sauer deutlicher zu hören ist - alle drei Ausführungsarten dieses Rhythmus bewegen sich allerdings völlig im Rahmen der auch heute noch üblichen Bandbreite von Spielweisen.

In einer Interpretationsanweisung gegen Ende des ersten Satzes zeigt sich Bülows Neigung zur lehrreichen »Vivisektion« der Musik besonders deutlich:

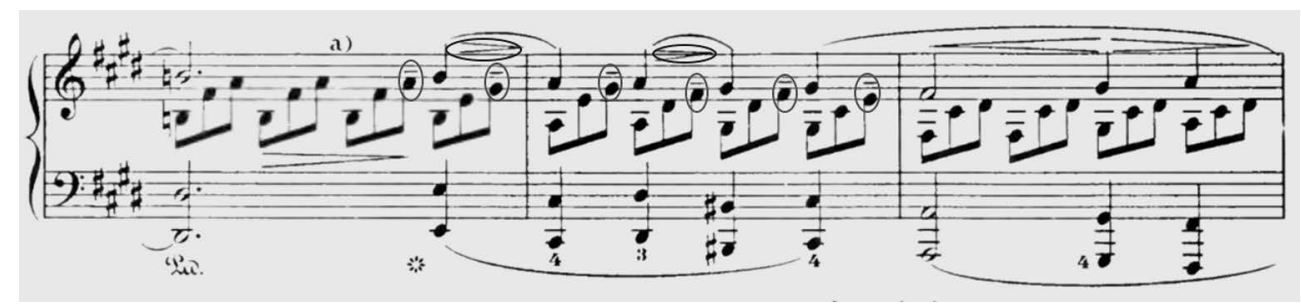

A B B IL D U N g 2 Beethoven: Sonate cis-Moll op. 27 Nr. 2, Bülow-Ausgabe, Takt 56-58

Die Striche über den jeweils letzten Achteln jeder Achtelgruppe ab a) markieren eine Innenstimme, die er durch Betonungen als Vorhalte herausgestellt wissen will. Außerdem fügt er kleine Decrescendi über der Oberstimme hinzu, sodass sich in dieser Modulation insgesamt vier unabhängig voneinander zu gestaltende Stimmen ergeben: Zusätzlich sind nämlich auch die Bassoktaven zu führen, welche ihre regulären Schwerpunkte auf der ersten und dritten Zählzeit haben, und schließlich noch die durchlaufende Achtelbegleitung im Altregister. Bülow ergänzt in seiner Fußnote dazu, dass alle Innenstimmen in diesem Satz aktiv gestaltet werden sollten - immer in Einklang mit dem Verlauf der Modulationen und den Gesetzen des »Schönklangs «. ${ }^{29}$

Carl Reinecke hob besonders das dialogisch-schlichte Spiel und die Vermeidung alles Scherzohaften in Franz Liszts Spiel des zweiten Satzes (Allegretto) hervor. ${ }^{30}$ In einer seiner seltenen außermusikalischen Charakterisierungen für ein Beethoven'sches Stück nannte Liszt diesen Satz einmal eine »Blume zwischen zwei Abgründen $«{ }^{3 \mathrm{I}}$ Dem formal wie atmosphärisch >einfachen`Satz zwischen den beiden berühmten Ecksätzen wird von den Pianistinnen und Pianisten und auch vom Publikum oft keine besondere Aufmerksamkeit geschenkt. Bezeichnend hierfür ist, dass auf der Schallplatte für Columbia

"[...] a utilization of the inner parts, in accordance with the laws of euphony and the course of the modulation «. Beethoven: Sonata quasi una fantasia op. 27/2, hg. von Hans von Bülow, in: Sonata Album, Book II, New York (Schirmer), S. I43-I60, hier S. I46. "[...] vermied auch Liszt Alles im Vortrage, was scherzo-artig anklingen konnte. Er spielte den Satz wie einen Dialog, der mit einer Frage beginnt, jeden scharfen Accent vermeidend.« Carl Reinecke: Die Beethoven'schen Clavier-Sonaten. Briefe an eine Freundin, Leipzig I897, S.48f., hier S. 49. 
Records von Arthur Friedheim überhaupt nur der erste und dritte Satz aufgenommen sind ${ }^{2}$ und auch Emil Sauer nur den ersten Satz einspielte.

Wilhelm von Lenz (I808-I883), Mitglied des kaiserlich-russischen Staatsrates und zugleich Musikschriftsteller, nahm als aktiver und ambitionierter Amateur I842 in Paris zunächst bei Liszt und dann bei Chopin Klavierunterricht. Er berichtet aus einer Stunde beim erst achtzehnjährigen Liszt folgende den zweiten Satz betreffende Details:

»L'allegretto de la sonate en ut dièse mineur qui remplace le scherzo me fournit l'occasion d'admirer la justesse des vues de Liszt en musique. Je venais de le lui jouer comme on écarte une petite difficulté quand une grande (le finale) vous attend. Liszt me demanda nonchalamment: >C'est facile, n'est-ce pas? J'avais dix-huit ans et répondis affirmativement. >Eh bien, non, dit Liszt, c'est un morceau sur lequel on passe sa vie quand on est artiste; les spondées suivis de dactyles sont les entrées des instruments à vent de l'orchestre du Conservatoire de Paris; le dessin coupé doit se piquer comme savent piquer les violons du Conservatoire. Et i il se mit à jouer le scherzo comme eût pu le jouer le merveilleux orchestre.«33

Diese Szene illustriert die große Bedeutung, die schon der junge Liszt dem kurzen Satz zumaß. Außerdem zeigt seine Behauptung, dieses Stück sei eines von jenen, nach dessen angemessener Realisierung ein wahrer Künstler sein ganzes Leben lang strebe, neben seinem hohen Anspruch an sich selbst zugleich auch eine gewisse jugendliche Altklugheit.

Immerhin folgten aber eine ganze Reihe von konkreten Interpretationshinweisen, die Lenz zu dieser pointierten Behauptung Liszts mit überliefert: Die Spondeen und darauffolgenden Daktylen des Themas - hier kommt vielleicht etwas von Liszts während der frühen Pariser Jahre autodidaktisch erlangter Belesenheit zum Ausdruck - solle man sich von den Bläsern gespielt vorstellen, während bei den darauffolgenden Staccati die Geigen des Conservatoires zum Einsatz kämen. Hier wird also deutlich, welch großen Wert schon der junge Liszt auf das orchestrale Klavierspiel legte. Dieses Merkmal der >Liszt-Schule spiegelt sich auch in mehreren Instruktionen der Bülow-Ausgabe wider (Abbildung 3).

Lenz erwähnt noch zwei kleinere Details: Auch die Oktaven der rechten Hand im Trio seien zu betonen, nicht nur die ganzen Noten (also die punktierte Halbe) der linken Hand. Die Bassfortschreitung im zweiten Teil des Trios sollte wie das Legato-Spiel zweier Celli klingen (Abbildung 4).

Bei letzterem Punkt kommt sogar noch das Thema des Fingersatzes zum Zuge: Lenz berichtet, wie Liszt ihm im Handumdrehen »le plus admirable doigter« für die Bindung

Außerdem scheint Friedheim die Aufnahme selbst verworfen zu haben, sie wurde nie in Amerika herausgebracht und bald ganz zurückgezogen; vgl. James Methuen-Campbell: Catalogue of Recordings by Classical Pianists, Bd. I, Oxfordshire I984. 


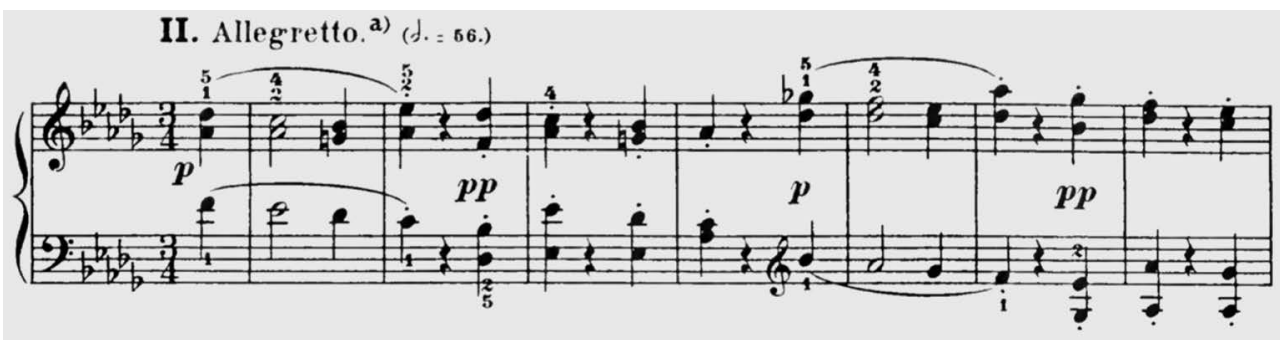

A в B ILdung 3 Bülow-Ausgabe, Takt 1-7

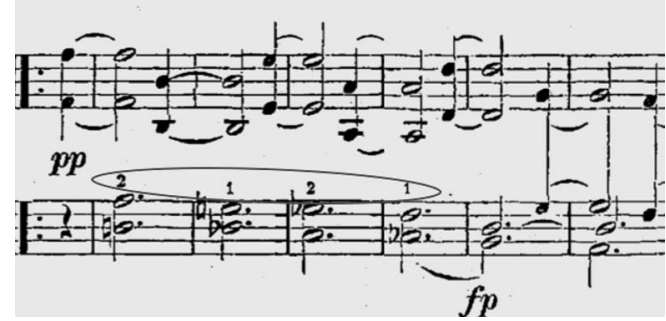

A в в I L D U G 4 Liszt-Ausgabe, Takt 45-50

dieser Bassquarten gegeben habe, nämlich 5-2/4-I/5-2/4-I. ${ }^{34}$ Dieser Fingersatz ist auch in der sonst so sparsam bezeichneten Liszt-Ausgabe angegeben, indem über den Doppelgriffen $2 / \mathrm{I} / 2 / \mathrm{I}$ notiert ist, was keine andere sinnvolle Fingerkombination zulässt als die ausführlich von Lenz beschriebene. Auch Bülow gibt hier einen Fingersatz an, allerdings konzentriert er sich offensichtlich auf die Bindung der unteren Leitstimme und lässt den Daumen nur mitrutschen: 2-I/3-I/4-I/5-I. Er hat also die Gleichwertigkeit der zwei >Celli weniger im Auge als Liszt.

In Lamonds Aufnahme dieses Satzes fällt vor allem das durchgängig asynchrone Spiel auf, welches den Unterschied zwischen erster Version des Themas (Akkorde zusammen) und der nachfolgenden Variation (Hände spielen Akkorde um Achtel versetzt) verschwimmen lässt. Die >Cello-Stelle kommt in seinem Spiel nicht besonders zum Tragen.

Um zur erwähnten Letterierung Liszts zurückzukehren: Interessanterweise hatte er im ersten und zweiten Satz der Mondscheinsonate keine Letterierung für notwendig gehalten, fügte sie aber im dritten Satz (Presto agitato) zur besseren Strukturierung wieder ein. Die ersten vierzehn Takte werden von keinem Gliederungsbuchstaben unterbrochen, obwohl man einen ersten Einschnitt durchaus in Takt 9 hätte erwarten können. Dies ist also ein Beispiel für einen längeren Sinnzusammenhang, der besonders im Vergleich mit der Kürze des nächsten Abschnitts auffällt: Liszt setzt die erste Unterteilung bei der Wiederholung der aufsteigenden Anfangspassage, die übernächste dann aber schon sechs Takte später bei Beginn des neuen Motivs. Dabei platziert er den 
Gliederungsbuchstaben spezifisch über der zweiten Viertel im Takt, denn die erste gehört noch als Abschluss zur vorherigen Passage:

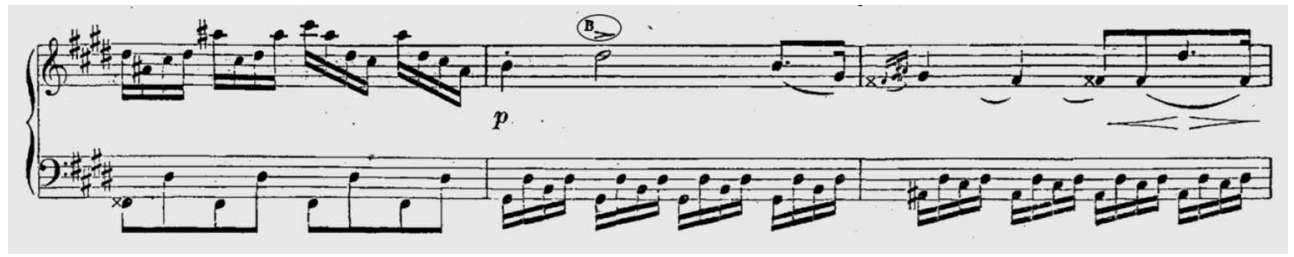

A в в ILdUNG 5 Liszt-Ausgabe, 3. Satz, Takt 20-22

Bülow gibt hier ebenfalls einen Gliederungshinweis, nämlich »ST « für Seitenthema, allerdings schon auf dem ersten Schlag. Er orientiert sich damit wohl eher an der linken Hand, die mit ihrer Begleitung in der neuen Tonart gis-Moll schon auf dem ersten Schlag einsetzt.

Ein Beispiel für eine weitere kurze und daher strukturell besonders bedeutungsvolle Passage in Liszts Gliederung folgt bald darauf:

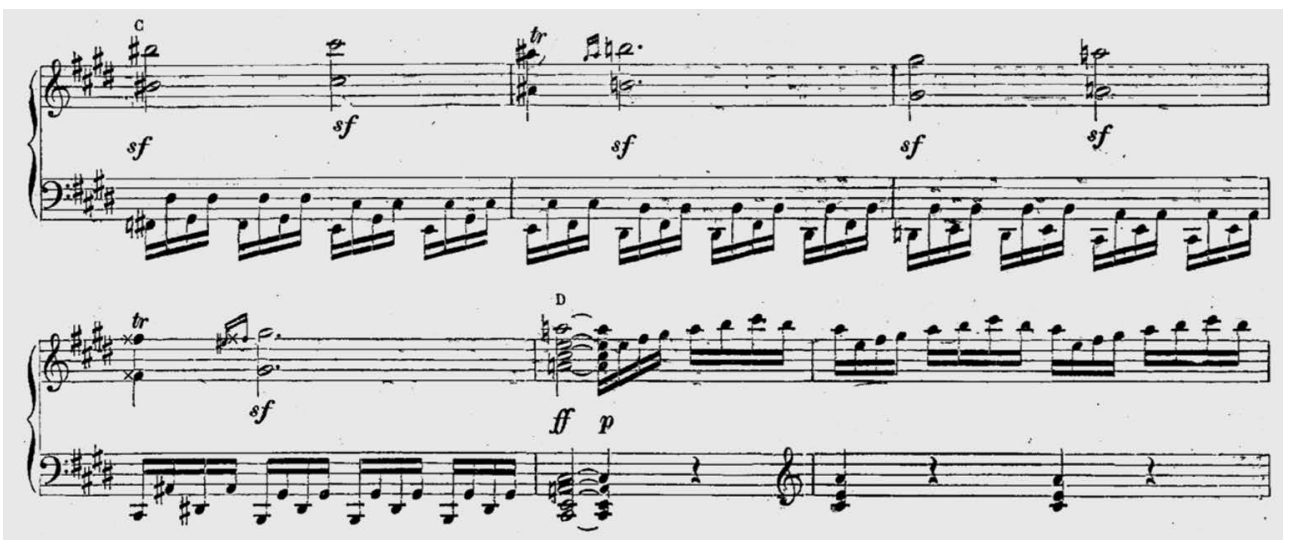

A в B ILdUNG 6 Liszt-Ausgabe, Takt 29-34

Die beiden zweitaktigen Sequenzen von C bis D modulieren schnell vom Tonfeld des Seitenthemas (gis-Moll) hin zur überraschend aufscheinenden neuen Tonart A-Dur, die für die nächsten zehn Takte vorherrscht. Das nächste musikalische Ereignis, das Staccato-Motiv, kehrt wieder zu gis-Moll zurück, von wo in die Schlussgruppe übergeleitet wird.

Bülow gibt zu den abgebildeten Takten auch eine Instruktion, allerdings bespricht er darin nur die Ausführung des Oktavtrillers, die er wegen des hohen Tempos sowie der nötigen Lautstärke als einfachen Doppelschlag auszuführen empfiehlt. Außerdem fügt er im Notentext Akzente auf jedem neuen Schritt der absteigenden Bassnoten hinzu. 
Um sich noch einmal die Individualität der Liszt'schen Gliederung je nach struktureller Bedeutung der Musik vor Augen zu führen: Vom Anfang des dritten Satzes bis zum ersten Gliederungspunkt A vergehen ganze vierzehn Takte, während Liszt für die Passage nach C nur vier Takte vergehen lässt, bevor er den nächsten Buchstaben D setzt.

Bülow schreibt zur späteren Variation des erwähnten Staccato-Motivs im Forte, dass ein Herunterhämmern dieser »leidenschaftlichen«Akkorde streng im Tempo ästhetisch offensichtlich falsch sei. Er empfiehlt stattdessen, die mit einem Sforzato beginnenden Takte (Takt 50 beziehungsweise 52) jeweils mit stärkerer Betonung und größerer Freiheit auszuspielen - auch um die rhythmische Wichtigkeit der zweiten Achtel herauszustellen - während die beiden Piano-Takte danach wieder zu beschleunigen seien:

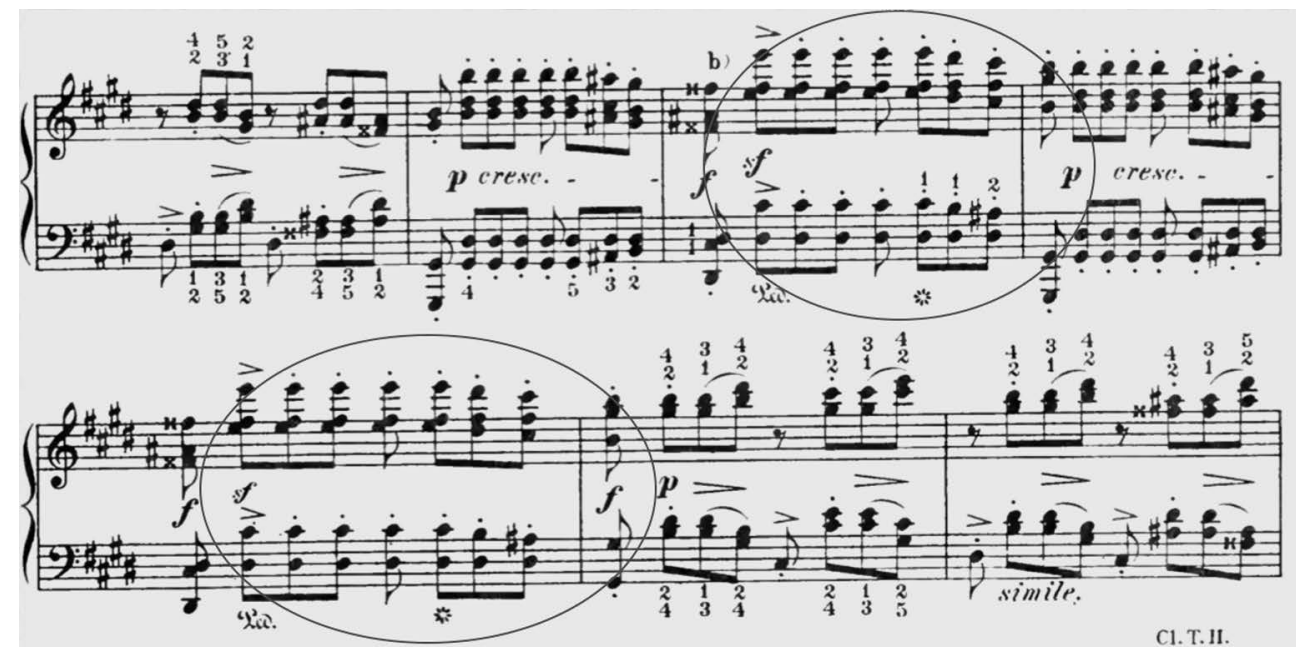

A в в ILDUNG 7 Bülow-Ausgabe, Takt 48-54

Diese ausführliche Beschreibung der wenigstens unter Liszt-Schülern selbstverständlichen Verbindung zwischen musikalischem Inhalt und Tempogestaltung ist ein Punkt, an dem sich Theorie und Praxis konkret vergleichen lassen: In der Aufnahme Frederic Lamonds von I926 ist gut nachzuvollziehen, wie er nicht nur diese Forte-Takte viel langsamer spielt, sondern sogar die von Bülow angegebene Pedalbenutzung befolgt möglicherweise kannte er Bülows Ansichten über die Sonate auch direkt aus dessen Unterricht. In Arthur Friedheims Aufnahme ist die gleiche Strategie zu beobachten, allerdings etwas weniger explizit; außerdem benutzt er das Pedal auch in den PianoTakten.

Die wenigen hier ausgewählten Stellen aus der Sonate können nur beispielhaft den noch weitgehend unerforschten Fundus von Hinweisen zu Interpretationsstrategien des Liszt-Kreises skizzieren: Eine genauere Untersuchung der Ausgabe von Liszt im Vergleich mit der Hans von Bülows und den Schüleraufnahmen wäre sicher sehr lohnend. 
Hans von Bülow als Advokat der Hammerklaviersonate Hans von Bülow setzte sich wie Liszt sehr für die Einführung der Hammerklaviersonate beim Publikum ein. Er spielte sie oft sogar zweimal hintereinander, um den Zuhörerinnen und Zuhörern die Möglichkeit zu geben, das komplexe Stück besser zu verstehen - eine wahre Tour de force für beide Seiten. 35 Auch für Aufführungen dieser Sonate ist seine Praxis des Präludierens belegt so leitete er sie im Jahr I879 beispielsweise mit einem Fragment der stimmungsverwandten Sonate op. 22 ein ${ }^{36}$ oder spielte zwischen Aufführungen der Sonate op. Ior und der Hammerklaviersonate Folgendes: 37

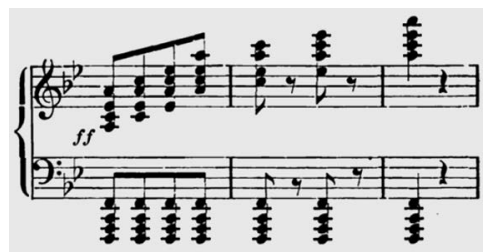

A B BILDUNG 8 Hans von Bülows Überleitung von der Sonate op. 101 zur Hammerklaviersonate

Über den Eindruck, den seine Interpretation beim Publikum im Allgemeinen hinterließ, ist allerdings wenig zu erfahren.

Sein Schüler Frederic Lamond jedoch wurde für sein Spiel gerade dieser Sonate sehr berühmt, und Bülow bescheinigte ihm schon bei den Frankfurter Meisterkursen (Sommer I884-I886), dass er »das Zeug zu diesem Stück« habe. ${ }^{8}$ Er stellte sich mit der Fuge daraus auch bei Franz Liszt in Weimar vor und die Hammerklaviersonate wurde später zu einem bewährten >Schlachtross< seiner Konzertprogramme. Hugo Riemann beschreibt, wie sogar die legendäre >Unspielbarkeit< des Stückes bei Lamond in Vergessenheit geriet:

\footnotetext{
»Lange hat das Werk als ein Prüfstein gegolten, ob ein Pianist imstande war, das Klavier zum Dröhnen und Brüllen zu bringen, bis Frederick Lamond durch eine ganz andere Art der Auffassung dem ein Ende gemacht hat. Er hat als erster es fertig gebracht, den Schein zu wecken, daß die Sonate überhaupt gar nicht schwer ist. Seine restlose Überwindung der technischen Schwierigkeiten hat die Linienführung der Thematik in den Vordergrund gestellt und das Interesse am klaren Aufbau sieghaft durchgeführt.«39
}

Edwin Fischer: Ludwig van Beethovens Klaviersonaten. Ein Begleiter für Studierende und Liebhaber, Wiesbaden 1956, S. II8.

36 »Der großen B dur-Sonate ließ Bülow den Beginn der in Miniatur stimmungsverwandten Op. 22 vorausklingen«, zit. nach Raab: »Zentralsonne der modernen Tonwelt«, S. I84.

37 José Vianna da Motta: Nachtrag zu Studien bei Hans von Bülow von Theodor Pfeiffer, Berlin/Leipzig I896, S. 82 .

38 Theodor Pfeiffer: Studien bei Hans von Bülow, Berlin 31894, S. 57 .

39 Hugo Riemann: Beethovens sämtliche Klavier-Solosonaten. Ästhetische und formal-technische Analyse mit historischen Notizen, Bd.3, Berlin 3[I920], S. 296. 
Leider existiert keine Aufnahme dieses Stückes von Lamond. Die früheste Gesamtaufnahme spielte Arthur Schnabel (I882-I95I) im Jahr I935 ein - er war allerdings kein Schüler von Liszt, sondern von Theodor Leschetitzky in Wien. Dennoch lohnt es sich, sein Herangehen an die Tempofrage zu beobachten, denn dies ist die früheste heute noch hörend nachzuvollziehende Umsetzung einer Interpretationshaltung, die nach den Berichten der Zeitgenossen auch für Liszt exemplarisch war: Die Wahrung der übergreifenden Architektur des Stückes, die besonders im ersten Satz nur in einem sehr schnellen Tempo möglich ist, da sich die harmonischen Verläufe trotz der raschen Notenwerte nur langsam entfalten. Zwar spielte Liszt vermutlich viel weniger falsche Noten als Schnabel, und sein konkretes Tempo lässt sich ebenfalls nicht mehr rekonstruieren. Schnabel bemüht sich aber mit seinen Mitteln um jenes Ideal, das neben der Darstellung der architektonischen Verhältnisse auch für Liszt im Vordergrund stand: den Geist der Komposition ungeachtet der technischen Schwierigkeiten zu übermitteln.

Für Schnabel scheint die »Überwindung der technischen Schwierigkeiten«, wie Riemann sie Lamond bescheinigte, gar nicht im Zentrum zu stehen, stattdessen scheint es ihm um eine möglichst sinnvolle Verwirklichung der extremen Originaltempi in den Ecksätzen zu gehen. Die technische Perfektion opfert er dabei seinem Verständnis der harmonischen Struktur und der Darstellung der formalen Zusammenhänge innerhalb dieser Sätze. Es scheint hier also wenig relevant, die Verwirklichung des Originaltempos im Sinne einer sportlich-pianistischen Herausforderung als »Schnabel gegen sich selbst« aufzufassen..$^{40}$ Vor dem Hintergrund des selbstverständlich nicht allein auf den Liszt-Kreis beschränkten Strebens nach architektonischer Klarheit drängt sich hingegen eine andere Sichtweise auf Schnabels Interpretation auf: seine Akzeptanz der zahlreichen pianistischen `Entgleisungen als zu vernachlässigendes Nebenprodukt eines strukturellen Imperativs.

Eine Stelle illustriert diesen Imperativ - Schnabels Fokus auf die zugrunde liegenden Strukturen zulasten der Details - besonders deutlich (Abbildung 9): Die drei verschieden intensiven Gelbstufen markieren den sich jeweils steigernden Grad des Vorwärtsdrängens an diesen zwei Stellen, der von Schnabel ohne Rücksicht auf Verluste (rote Kreise weisen auf besonders auffälliges >Verschlucken wird. In der ersten Stelle kommt es nach dem Erreichen des Spitzentons g" in einer dritten Sequenz zur Auflösung nach Es-Dur, was entsprechend der satztechnischen •Entspannung nach einer ungebremsten Umsetzung verlangt. Dies ist allerdings aus pianistischer Sicht besonders schwierig, da das rasche Tempo, das bereits in den vorherigen 


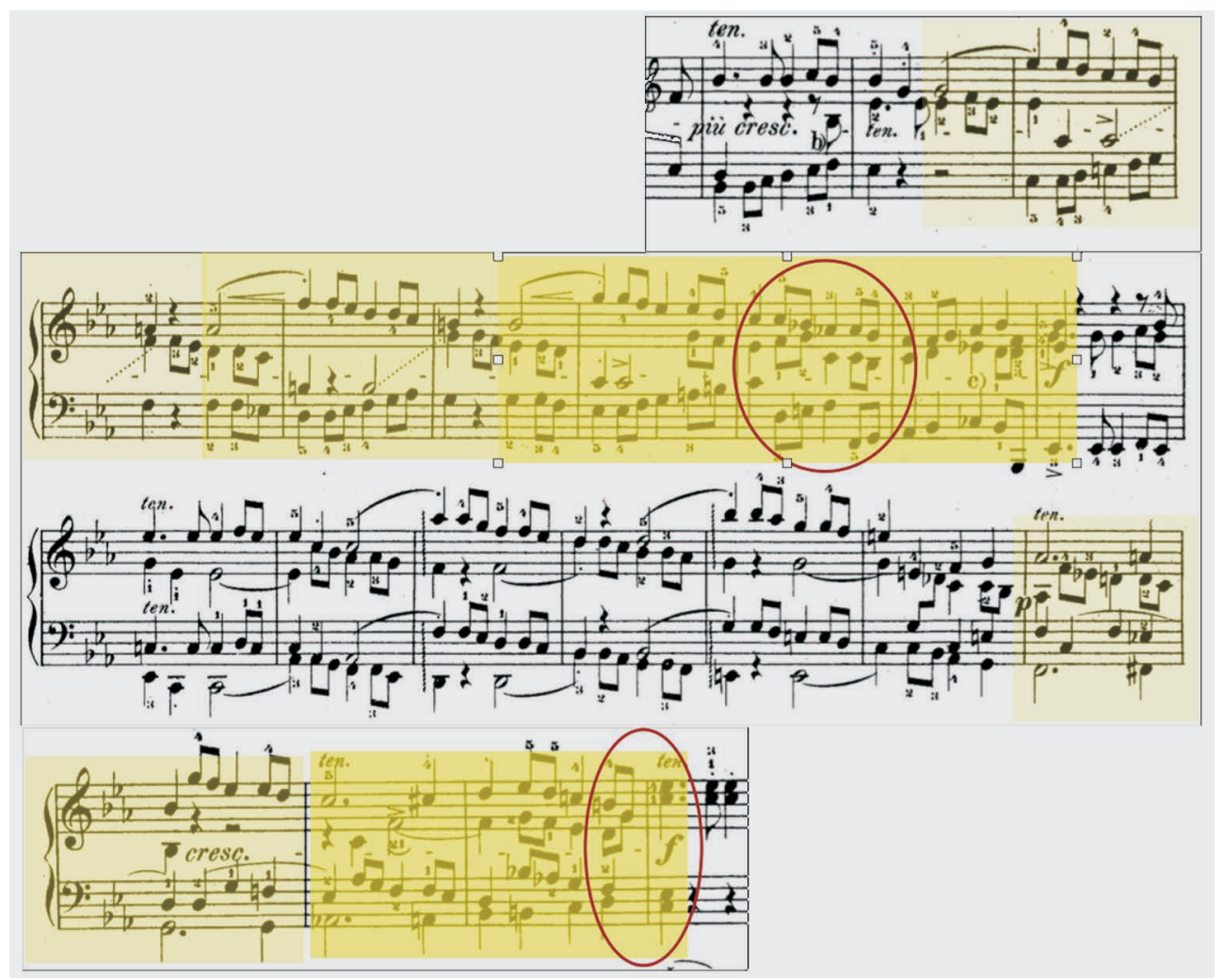

A B B ILDUNG 9 Bülow-Ausgabe der Hammerklaviersonate,

Durchführung 1. Satz, Takt 147-167

Takten kaum zu meistern war, nun mit den Achtel-Doppelgriffen vollends unmöglich wird. Statt aber zu verlangsamen, um alle Griffe ausführen zu können, verschluckt Schnabel alles, was der Wahrung des inhaltlichen Schwungs im Wege steht. Die gleiche Tendenz ist an der zweiten markierten Stelle zu beobachten, die noch um ein Sequenzglied verkürzt ist - im Sinne der allgemeinen Steigerung nun in noch extremerem Maße.

Die drei einleitenden Instruktionen Bülows zum ersten Satz sind alle besonders interessant. Zunächst liefert er eine ausführliche Erklärung, weshalb das Originaltempo, das er als lediglich von Carl Czerny angegeben bezeichnet, obwohl es tatsächlich von Beethoven selbst stammt, zu schnell sei:

"Das Czernysche Tempo d = I38, das zu der wuchtigen Energie des Thema so wenig stimmt und selbst für die einer grösseren Beschleunigung fähigen Abschnitte dieses Satzes zu rasch gegriffen erscheint, findet vielleicht in der Klanglosigkeit der damaligen Wiener Klaviere eine Art Rechtfertigung. Auf einem heutigen Conzertflügel erster Qualität [...] würde das Czernysche Tempo verwirrend und verwischend wirken. ${ }^{4}$

41 Bülow-Ausgabe, S. 23. 
Er setzt es in seiner Ausgabe auf Halbe = II2 herab. Dies scheint im Gegensatz zu Franz Liszts strenger Haltung gegenüber Interpretationen zu stehen, die auf die Schwierigkeiten der pianistischen Umsetzung Rücksicht nehmen ${ }^{42}$ und dem Satz einen Maestoso-Charakter verleihen, aber wie bereits oben erwähnt, ist das absolute Tempo der Liszt'schen Interpretation dadurch natürlich nicht ermittelbar.

Der zweite Kommentar Bülows bezieht sich auf das »löwenhafte Anspringen«, 43 den weiten Eröffnungssprung der linken Hand, der angeblich schon den jungen Liszt besonders fasziniert hatte: »Namentlich der Baßsprung b-d und dann auff entzückte mich aufs höchste.«44 Bülow gibt hier eine einfachere Alternative mit zwei Händen an, wozu er ausführt:

»Spieler, welchen der schwierige Sprung der linken Hand unter kräftigster Betonung des AuftaktAchtels und Vermeidung jeder hier unangemessenen Zögerung nicht gelingen sollte, werden wohlthun, beide Hände mit einander abwechseln zu lassen«.

In der dritten Instruktion kommt das besprochene symphonische Denken Bülows, welches so typisch für den ganzen Liszt-Kreis ist, zum Ausdruck. Um zu entscheiden, wie man den Griff bei zu kleiner Spannweite der Hand adaptieren kann, entwirft er eine mögliche Orchestrierung:

»Man denke sich die Stelle orchestrirt:[ Geigen und Flöten würden es"' d"' zuertheilt erhalten; d" ist aber jedenfalls für Naturtrompete gedacht und deren Charakter vollkommen entsprechend.«45

Also dürfe das d" im Daumen keinesfalls fallengelassen werden, sondern lieber der zweite Finger, der das f" greift. Aus Bülows orchestralem Denken ergibt sich also, dass dieser Ton hier eine weniger zentrale Rolle spielt und deswegen notfalls ausgelassen werden darf.

Eine weitere berühmte Instruktion Bülows zum ersten Satz behandelt eine Unklarheit im Notentext - es geht um den bis heute diskutierten Streitfall einer harmonischen Deutung in der Überleitung zur Reprise: Je nach Lesart kann man in Takt 224 den erwarteten Leitton a (das explizite Auflösungszeichen fehlt allerdings in der Erstausgabe) oder stattdessen ais als eine extravagante enharmonische Vorwegnahme des Grundtons lesen. Liszt nimmt in seiner Ausgabe ebenfalls ais an, während Frederic Lamond $a$ ab-

42 Vgl. beispielsweise in den Memoiren des Schülers William Mason: Memories of a Musical Life, New York I900, S. Io3f.

43 Fischer: Ludwig van Beethovens Klaviersonaten, S. II9.

44 August Göllerich: Franz Liszt, Berlin 1908, S. I59f. Auch dieses angebliche Zitat Liszts wird von Schröter dem Aufbau eines Beethoven-Mythos um Liszt, in diesem Fall durch Göllerich, zugeschrieben, da er als Grundlage für diese Äußerung den seiner Meinung nach unglaubwürdigen Brief Liszts an die Fürstin vermutet; vgl. Schröter: »Der Name Beethoven ist heilig in der Kunst«, S. I4. 
druckt, aber in einer lapidaren Fußnote dazu schreibt, Bülow spiele ais. Hans von Bülows langer Text zu dieser Stelle beginnt mit folgenden, oft zitierten Worten:

»Die Beethovenverbesserer früherer Zeit [...] hatten das rais $<$ in den folgenden beiden Takten in sa umgewandelt, somit die enharmonische Genialität zu einer chromatischen Trivialität erniedrigt.« ${ }^{46}$

Interessanterweise trennte sich Bülow später während seiner Frankfurter Meisterkurse ohne Aufhebens von seiner vorher so streitbar vertretenen Auffassung:

»Ein Bekannter von mir hat im Original das Auflösungszeichen vor dem ais ganz verblasst entdeckt. Als ich meine Beethoven-Ausgabe machte, wusste ich das nicht, glaubte analoge Stellen in anderen Werken des Meisters gefunden zu haben und plaidirte für ais. Jetzt bitte ich Sie aber a zu spielen.«47

Eine Originalausgabe, in der man verblasste Auflösungszeichen hätte entdecken können, ist von dieser Sonate allerdings nie aufgetaucht. Bis heute sind beide Lesarten zu hören.

In der Ausgabe Eugen d'Alberts finden sich direkt im Notentext zahlreiche Vortragsbezeichnungen, die sowohl den Ausdrucksgehalt wie das Tempo betreffen. Beim Eintritt des Seitenthemas in der linken Hand (Takt 62) gibt er beispielsweise più tranquillo an und stimmt dort also inhaltlich mit Bülows ausformulierter Instruktion überein: »Die Bewegung dürfte hierbei wohl eine unwesentliche Abnahme vertragen. ¿48 $^{8}$ In der Schlussgruppe der Exposition schreibt er animato und »Erheblich rascher, mit feurigem Aufschwung «49 - dies deckt sich mit seiner persönlichen Spielpraxis.

Zum dritten Satz, dem Adagio sostenuto, schreibt Bülow Folgendes:

»Zu fast keinem Tonstücke des Meisters wird eine so andächtige, ehrfurchtsvolle Hingebung erfordert, um seiner schmerzreichen Erhabenheit gerecht zu werden. Hier hört das Klavierspielen auf: wer auf seinem Instrumente nicht seelenvoll zu >sprechen vermag, begnüge sich mit dem Lesen.«50

Von modernen Pianistinnen und Pianisten wird der Satz oft viel langsamer gespielt als Beethovens Metronomangabe Achtel = 92, beispielsweise von den berühmten Beethoven-Interpreten Claudio Arrau oder Solomon (Solomon Cutner). Bülow hingegen hält das angegebene Tempo offenbar für langsam genug und auch Lamond und d'Albert übernehmen diese originale Metronomzahl ohne weiteren Kommentar. Bülow schreibt in seiner Instruktion sogar noch dazu: »Bei Klavieren von grosser Klangfülle kann das Zeitmaass noch [sic] langsamer genommen werden. ${ }^{\mathrm{I}}$ Liszt hingegen stufte das Tempo in seiner Ausgabe zu Achtel $=84$ herab.

46 Bülow-Ausgabe, S. 30.

47 Vianna da Motta: Nachtrag, S. 43 f.

48 Bülow-Ausgabe, S. 25.

49 Ludwig van Beethoven: Sonate Nr. 29 B-Dur. Grosse Sonate für das Hammerklavier, hg. von Eugen d'Albert, Leipzig [1903], S. 6.

50 Bülow-Ausgabe, S. 42 .

51 Ebd. 
Zu der berühmten Kontraststelle in G-Dur gibt Bülow zwei Instruktionen, die exemplarisch für seine Editionsästhetik sind:

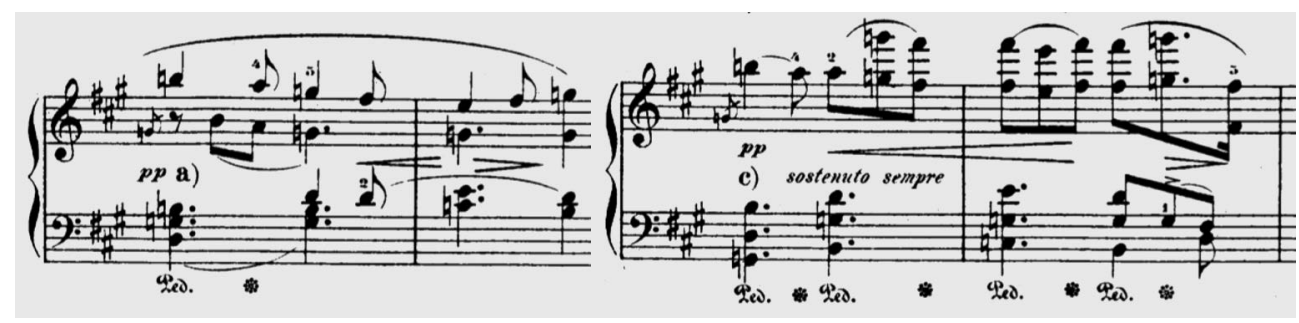

A в в I L D U G 10 Bülow-Ausgabe, Takt 14/15 und 22/23

Zum ersten Auftritt dieses Motivs schreibt er:

»Das >d des Basses ist nicht als Grundton eines Quartsextaccordes aufzufassen, sondern als eine Verschleierung des für das Halbdunkel der Empfindung allzu entschiedenen Dreiklanges von G dur. Man vergleiche hiermit den Eintritt des Themas im Adagio des Streichquartetts Op. I27. Die Bassführung in Beethovens späteren Werken ist überhaupt nie vom Standpunkte der Harmonik, sondern vom contrapunktischen (dem der Melodie) ins Auge zu fassen.»

Und zu den anderen beiden Takten: »Ein Arpeggieren dieser Akkorde in der Dezimenlage ist nach Kräften zu vermeiden: Die Klangfärbung muss dem Charakter eines Pianissimo von Posaunen entsprechen. ( $^{2}$

Hier begegnet man gleich mehreren in der Einleitung zu diesem Kapitel aufgezählten Kategorien, in die Bülows Kommentare eingeteilt wurden: Zunächst gibt er einen ästhetischen Hinweis zur Rolle des Basses mit Querverweis auf ein anderes Stück Beethovens (Streichquartett op. I27). Darauf folgt eine Ausweitung des Gesagten auf die Bassführung in Beethoven-Werken im Allgemeinen. Der zweite Kommentar verbindet eine praktisch-pianistische Instruktion (hier sei kein Arpeggieren angebracht) mit einer musikalischen Erläuterung, in diesem Fall wieder einer ziemlich exotischen Orchestrierung (ein Pianissimo von Posaunen). Hier wird die ganze Bandbreite von Bülows Herangehensweise an die Musik offenbar - und mit dem Querverweis auch sein Ehrgeiz, den Horizont der Leserin, des Lesers über das Verständnis der vorliegenden Sonate hinaus zu erweitern. Am Ende des Finales steht schließlich ein direkter Hinweis Bülows auf eine alternative Spielweise Liszts (Abbildung II).

Die »moderne Umschreibung «betrifft also die eigentlichen Triller, die so klarer und stärker herausgebracht werden können. Was allerdings von Bülow nicht kommentiert, aber noch interessanter ist: der im Kleinstich mitabgedruckte Takt vor den Trillern, dessen Abbildung für die Instruktion nicht nötig gewesen wäre, aber eine signifikante 

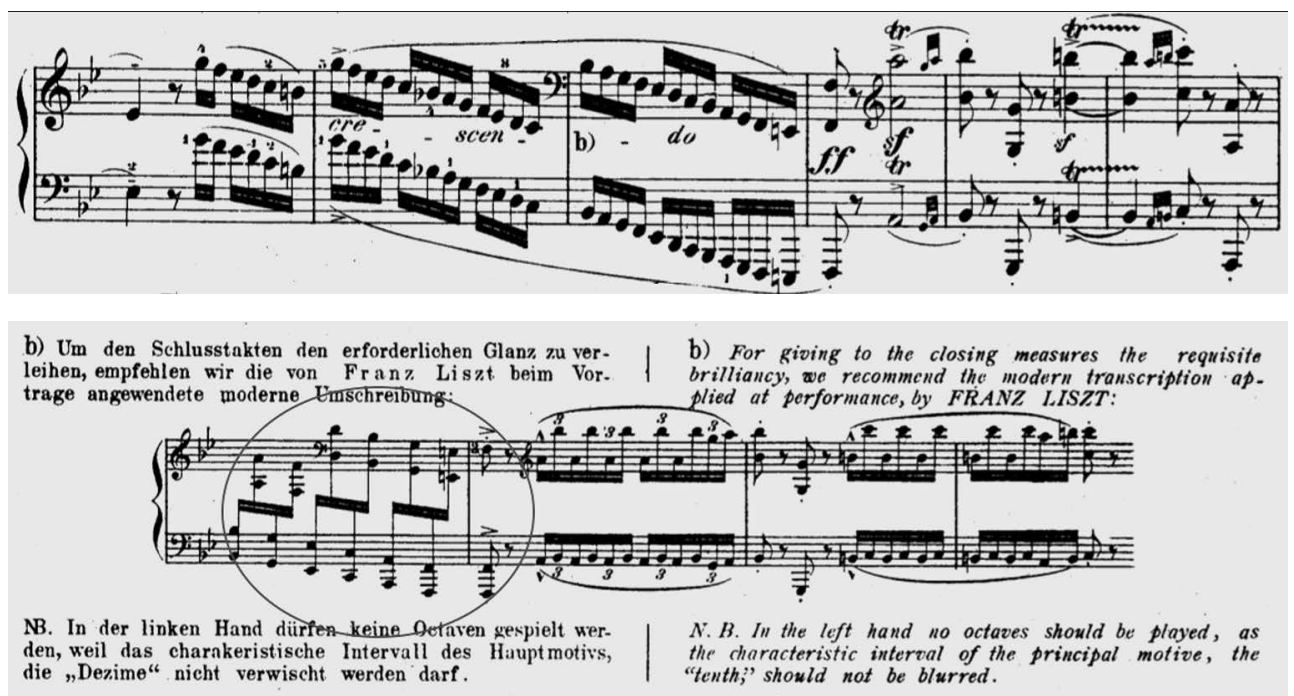

A в в IL D U N G 1 i Bülow-Ausgabe, 4. Satz, Takt 395-400, mit Anmerkung

Änderung des Textes zeigt. Statt der originalen beidhändigen Sechzehnteltonleiter schreibt Bülow hier eine alternierende Oktaven-Kaskade. Dies ist eine aus anderen Quellen bekannte Spielweise Liszts, die dieser in seiner >Glanzzeit< besonders in Opernfantasien und anderen Virtuosenstücken anwandte. Amy Fay beispielsweise beschreibt eine Präsentation dieser Spielweise durch Liszt im Jahr I873:

»Oh, I've invented a great many things, < said he, indifferently - >this, for instance, $<$ - and he began playing a double roll of octaves in chromatics in the bass of the piano. It was very grand, and made the room reverberate. 453

Wie das Zitat zeigt, zeichnen sich diese alternierenden Oktaven besonders durch große Lautstärke und (eine etwas grobe) »Großartigkeit« aus. Beides ist am Ende der Fuge im Allgemeinen zwar durchaus angebracht, aber doch auf einer ganz anderen Stilebene: Während die Liszt'sche Variante der Oktaven-Triller tatsächlich eine Verstärkung der Klangfülle ermöglicht, ohne jedoch eine allzu aufdringliche Virtuosität hinzuzufügen, müsste man die alternierenden Oktaven anstelle der originalen Unisono-Tonleiter wohl mit dem Begriff >Effekthascherei b belegen.

$\mathrm{Da}$ Wendelin Weissheimer ${ }^{54}$ allerdings von zahlreichen Verdoppelungen in der Fuge sprach, kann man sich durchaus vorstellen, dass Liszt seine »Erfindung« an dieser Stelle wirklich einsetzte. Darüber hinaus kann man aber sicher auch davon ausgehen, dass Liszt sich an das jeweilige Publikum anpasste - also andere Stilmittel vor den Ohren

53 Fay: Music-Study in Germany, S. 24I.

54 Vgl. Wendelin Weißheimer: Erlebnisse mit Franz Liszt, Richard Wagner und vielen anderen Zeitgenossen, Stuttgart/Leipzig I898, S. 20. 
eines staunenden Kreises junger Männer einsetzte als beispielsweise in der berühmten Aufführung I836 in Paris, von der Hector Berlioz behauptete:»Pas une note n'a été omise, pas une note n'a été ajoutée (je suivais des yeux la partition)«.55

Hans von Bülow war eine facettenreiche Persönlichkeit ganz eigener Prägung. Es gilt also zwischen Elementen in seiner Ausgabe zu unterscheiden, die nur für seine eigene Beethoven-Vermittlung charakteristisch sind und solchen, die wirklich als auf Liszt und die »Frucht seiner Lehre «zurückgehend identifiziert werden können. Zu letzteren gehört - neben den direkten Erwähnungen Liszts - vor allem das Streben danach, die formalen Zusammenhänge und die kompositorische Struktur jeder Sonate genau zu verstehen: So wird in Berichten immer wieder hervorgehoben, dass Liszts Interpretationen auch dadurch frappierten, dass sie klangen, als ob er der Komponist des jeweiligen Stückes sei. Bülow realisiert dieses übernommene Ideal allerdings in seiner ganz eigenen, im Gegensatz zu Liszt von den Zeitgenossen oft als penetrant empfundenen Weise. Liszt kehrte diesen Aspekt in einem Kommentar wie gewöhnlich ins Positive: »Bülow ist ein Schulmeister, aber ein vornehmer. ${ }^{6}{ }^{6}$

Zu diesem Thema gehört auch ein exzessiv auf Ordnung und Konsistenz achtender Persönlichkeitszug Bülows, welcher natürlich völlig unabhängig von Liszts Lehren existierte. Er ist beispielsweise in der genauen Festlegung von Fermatenlängen greifbar - so am Anfang der Sonaten op. 53 und Io6 - die dem als besonders spontan und rhetorisch angelegten Spiel Liszts sicher gar nicht entsprachen.

Eine andere durchgängig zu beobachtende Gemeinsamkeit war die Strategie, sich stets Orchestrierungen des Klaviersatzes zu überlegen, um ein befriedigendes Klangresultat zu erreichen.

Weiterhin lassen sich eine Reihe von wiederkehrenden Einzeldetails auf Liszt zurückführen: Dazu gehört die Neigung, Einzelstimmen in dynamisch mächtigen Stellen zu Oktaven zu verdoppeln. Auch die Bereitschaft, in einem Zeitalter unzähliger Regeln für eine >richtige $<$ Handhaltung und einen korrekten Fingersatz im Gegenteil ganz unorthodoxe Fingersätze zu benutzen, um eine bestimmte Textur oder Artikulation herauszubringen, zählt unter solche übernommene Grundsätze aus Liszts Unterricht - bei Bülow beispielsweise im ersten Satz der Hammerklaviersonate am Fingersatz »à la Chopin« in Takt 74 zu sehen. 57

Ein nicht nur bei >Lisztianern zu beobachtendes, aber bei Liszt und Bülow besonders zentrales Charakteristikum war der grundlegende Wandel ihrer künstlerischen Ideale im Laufe des Lebens, der sich für das Definieren einer bestimmten Interpreta- 
tionshaltung als Hindernis erweist. Während Liszt, wie oben dargestellt, vom »Charla$\tan \ll^{8}$ zum >pietätvollen< Propheten Beethovens wurde, wandte sich Bülow ganz vom Liszt-Kreis ab und verlegte auch seinen Fokus auf andere Beethoven-Werke als die von Liszt besonders häufig gespielten. Selbst Bülows Haltung zu seiner eigenen instruktiven Edition änderte sich. So sagte er während seiner Frankfurter Meisterkursen zu einem Schüler:

»Es ist sehr schmeichelhaft für mich, dass Sie meine Ausgabe bringen, ich rathe Ihnen aber doch, Klindworth's Ausgabe zu nehmen; dort finden Sie all das Gute meiner Ausgabe, das Ueberflüssige ausgeschieden, das Irrige verbessert. 259

58 So wurde beispielsweise die Eröffnung eines Besuchs, den Liszt bei einem musikinteressierten Maler namens Bonaventure Laurens in Montpellier I844 machte, folgendermaßen vom Bruder des Malers festgehalten: »Laurens, der von Liszts >Klavier-Akrobatikı gehört hatte, begrüßte ihn wenig höflich: ,Sie gelten für einen ebenso großen Charlatan wie großen Künstler.« Vgl. Marcelle Hermann: J.-J. B. Laurens' Beziehungen zu deutschen Musikern, in: Schweizerische Musikzeitung I05 (I965), S. 257-266, hier S. 260.

59 Da Motta: Nachträge, S.3r. 


\section{Inhalt}

Vorwort 8

INTERPRETATION - BEGRIFF, METHODE, PRAXIS

Laure Spaltenstein Interpretation als treue Übersetzung. Zur Frühgeschichte eines vieldeutigen Begriffs I5

Kai Köpp Von der Quelle zur Methode. Zum Entwurf einer historischen Interpretationsforschung $\quad 28$

Manuel Bärtsch >Interpretation‘. Beethovens Sonate A-Dur op. IOI in der Sicht von Eugen d'Albert und Frederic Lamond

Sebastian Bausch Klavierrollen als Interpretationsdokumente. Ein Erfahrungsbericht als Leitfaden für Einsteiger $\quad 7 \mathrm{I}$

Camilla Köhnken Beethoven-Auslegung zwischen Liszts »Deklamationsstil« und Bülows »Vivisektionsversuchen«. Auf den Spuren Liszt'scher Interpretationsideale in Hans von Bülows instruktiver Edition der Klaviersonaten Beethovens $\quad 92$

Neal Peres Da Costa Carl Reinecke's Performance of his Arrangement of the Second Movement from Mozart's Piano Concerto K. 488. Some Thoughts on Style and the Hidden Messages in Musical Notation

II4

Carolina Estrada Bascuñana Enrique Granados's Performance Style.

Visualising the Audible Evidence I5O

Lukas Näf Tempogestaltung in Weberns Sinfonie op. 2I I80

INTERPRETATION - AUFFÜHRUNGSGESCHICHTE

Christoph Moor "Ein so erklärtes Lieblingsstück der hiesigen Kunstfreunde«. Die Rezeptionsgeschichte der Jupiter-Sinfonie in Beethovens Wien

Luisa Klaus Objektive Bruckner-Interpretation? Zur Aufführung der Trio-Entwürfe für die Neunte Sinfonie I940 205

Chris Walton Von innen und von außen. Beethovens Neunte Sinfonie und die $>$ Wagner'sche< Dirigiertradition $\quad 2 \mathrm{I} 8$

Lena-Lisa Wüstendörfer Streit um Fidelio. Gustav Mahler und Felix Weingartner im Disput um Werktreue $\quad 238$

INTERMEZZO

Robert Levin Turning Point to Musical Modernity. Beethoven as Executor of the Legacy of C. P. E. Bach. Concert Lecture 249

INTERPRETATION - INSTRUMENTE, ANALYSE, EDITION

Martin Skamletz «Man hat diese Erweiterung des Tonumfanges seit ein paar Jahren an den Tasteninstrumenten sehr weit getrieben.« Der Umgang mit Grenzen beim späten Mozart und beim frühen Beethoven $\quad 263$ 
Stephan Zirwes Analyse und Interpretation. Adolph Bernhard Marx' Beethoven-Analysen 29I

Michael Ladenburger Was können wir aus Originalhandschriften von Beethoven für eine angemessene Interpretation lernen? 30I

Federica Rovelli Die Skizzenbuch-Ausgaben und ihre mögliche digitale Zukunft 3 I7

Johannes Gebauer Interpretationspraktische Stemmatik. Philologische Methoden in der Interpretationsforschung am Beispiel annotierter Notenausgaben von Rodes 24 Capricen und Beethovens Violinkonzert

John Rink Chopin Copying Chopin 349

Tomasz Herbut Alexander Goldenweiser und Beethovens Sonate op. IIo- eine Spurensuche 366

INTERPRETATION - KREATIVE ANEIGNUNG

Thomas Gartmann Beethoven als sein eigener Interpret. Gedanken zur Bearbeitung der Klaviersonate op. I4/ז für Streichquartett

Ivo Haag Die Sinfonien von Johannes Brahms - (auch) Klaviermusik?

Michael Lehner Das Orchester auf dem Klavier. Welte-Klavierrollen von Gustav Mahler und Richard Strauss als interpretationsanalytische Quellen

Roger Allen “That Is What Music Really Is". Richard Wagner's Reception of Beethoven's Piano Sonata in A Major Op. IOI $43 \mathrm{I}$

Daniel Allenbach Eine heroische< Neunte? Dmitri Schostakowitschs Neunte Sinfonie im Vergleich mit Ludwig van Beethoenvs Sinfonien Nr.3 und 9 44I

Simeon Thompson Beethoven und der Zweite Weltkrieg in der künstlerischen Reflexion der Nachkriegszeit. Stanley Kubricks A Clockwork Orange und Rolf Liebermanns Leonore 40/45 456

Michelle Ziegler Rettungsversuch im Jubiläumsjahr. Mauricio Kagels Aufarbeitung der Beethoven-Rezeption in der Ludwig van-Werkgruppe (I970) 465

Leo Dick Über den späten Beethoven zur >Postidentität،. Die Suche nach liminalen Räumen im gegenwärtigen Musiktheater am Beispiel von Matthias Rebstocks Berliner Produktion Büro für postidentisches Leben $\quad 476$

Elizabeth Waterhouse Choreographic Re-mix. William Forsythe's Trio (I996) and Beethoven's String Quartet No. I5 in a Minor Op. I32 487

László Stachó "Gradus ad Parnassum".

The Purgatory of Instrumental Technique

Namen-, Werk- und Ortsregister 522

Die Autorinnen und Autoren der Beiträge 


\section{Rund um BeEthoven \\ Interpretationsforschung heute • \\ Herausgegeben von Thomas}

Gartmann und Daniel Allenbach 


\section{MUSIKFORSCHUNG DER Hochschule der KÜnste Bern Herausgegeben von Martin Skamletz und Thomas Gartmann Band 14}


0 Dieses Buch ist in gedruckter Form im Dezember 2019 in erster Auflage in der Edition Argus in Schliengen/Markgräflerland erschienen. Gestaltet und gesetzt wurde es im Verlag aus der Seria und der SeriaSans, die von Martin Majoor im Jahre 2000 gezeichnet wurden. Gedruckt wurde es auf Eos, einem holzfreien, säurefreien, chlorfreien und alterungsbeständigen Werkdruckpapier der Papierfabrik Salzer im niederösterreichischen Sankt Pölten. Das Vorsatzpapier Caribic cherry wurde von Igepa in Hamburg geliefert. Rives Tradition, ein Recyclingpapier mit leichter Filznarbung, das für den Bezug des Umschlags verwendet wurde, stellt die Papierfabrik Arjo Wiggins in Issy-les-Moulineaux bei Paris her. Das Kapitalband mit rot-schwarzer Raupe lieferte die Firma Dr. Günther Kast aus Sonthofen im Oberallgäu, die auf technische Gewebe und Spezialfasererzeugnisse spezialisiert ist. Gedruckt und gebunden wurde das Buch von der Firma Bookstation im bayerischen Anzing. Im Internet finden Sie Informationen über das gesamte Verlagsprogramm unter www.editionargus.de, zum Institut Interpretation der Hochschule der Künste Bern unter www.hkb.bfh.ch/interpretation und www.hkb-interpretation.ch. Die Deutsche Nationalbibliothek verzeichnet diese Publikation in der Deutschen Nationalbibliografie; detaillierte bibliografische Daten sind im Internet über www.dnb.de abrufbar. (c) der zeitgleich erschienenen digitalen Version: die Autorinnen und Autoren, 20I9. Dieses Werk ist lizenziert unter einer Creative Commons Namensnennung-Nicht kommerziell 4.0 International Lizenz (CC BY-NC 4.o). DoI: https://doi.org/I0.26045/kp64-6I78 ISBN 978-3-93I264-94-9 